\title{
Mesenchymal cells regulate the response of acute lymphoblastic leukemia cells to asparaginase
}

\author{
Shotaro Iwamoto, ${ }^{1}$ Keichiro Mihara, ${ }^{1}$ James R. Downing, ${ }^{2,3}$ \\ Ching-Hon Pui, 1,2,3 and Dario Campana ${ }^{1,2,3}$ \\ 1Department of Oncology and 2Department of Pathology, St. Jude Children's Research Hospital, Memphis, Tennessee, USA. \\ 3 University of Tennessee College of Medicine, Memphis, Tennessee, USA.
}

\begin{abstract}
Because of their low asparagine synthetase (ASNS) expression and asparagine biosynthesis, acute lymphoblastic leukemia (ALL) cells are exquisitely sensitive to asparagine depletion. Consequently, asparaginase is a major component of ALL therapy, but the mechanisms regulating the susceptibility of leukemic cells to this agent are unclear. In 288 children with ALL, cellular ASNS expression was more likely to be high in T-lineage ALL and low in B-lineage ALL with TEL-AML1 or hyperdiploidy. However, ASNS expression levels in bone marrow-derived mesenchymal cells (MSCs), which form the microenvironment where leukemic cells grow, were on average 20 times higher than those in ALL cells. MSCs protected ALL cells from asparaginase cytotoxicity in coculture experiments. This protective effect correlated with levels of ASNS expression: downregulation by RNA interference decreased the capacity of MSCs to protect ALL cells from asparaginase, whereas enforced ASNS expression conferred enhanced protection. Asparagine secretion by MSCs was directly related to their ASNS expression levels, suggesting a mechanism - increased concentrations of asparagine in the leukemic cell microenvironment - for the protective effects we observed. These results provide what we believe to be a new basis for understanding asparaginase resistance in ALL and indicate that MSC niches in the bone marrow can form a safe haven for leukemic cells.
\end{abstract}

\section{Introduction}

Asparaginase is a universally used component of childhood acute lymphoblastic leukemia (ALL) treatments, in both the remission induction phase and the postinduction period (1-10). The critical role of asparaginase in the context of multiagent chemotherapy has been amply demonstrated by clinical studies showing direct relations between treatment outcome and dose intensity of asparaginase (11) and between an inferior outcome and the use of an asparaginase preparation with a shorter half-life (12). A recent randomized study showed a significant improvement in eventfree and overall survival for children with ALL who were assigned to receive high-dose asparaginase during continuation therapy compared with those who did not receive asparaginase (13).

The mechanism underlying the antileukemic activity of asparaginase relies on the conversion of asparagine and glutamine to aspartate and glutamate, respectively $(1,14-16)$. Leukemic lymphoblasts are exquisitely sensitive to the depletion of exogenous asparagine and glutamine because their capacity to produce their own asparagine supply is exceedingly low, owing to their generally low expression of the asparagine synthetase (ASNS) gene $(1,14,15,17-20)$. In some experimental models of leukemia, cellular levels of ASNS were directly related to the resistance of leukemic cells to Asparaginase $(17,19,20)$, suggesting that measurements of this enzyme in ALL cells from patients might be used as a predictor of leukemic cell sensitivity to asparaginase.

Nonstandard abbreviations used: ALL, acute lymphoblastic leukemia; ASNS, asparagine synthetase; MSC, mesenchymal cell; TBP, TATA box-binding protein; TERT telomerase reverse transcriptase.

Conflict of interest: The authors have declared that no conflict of interest exists. Citation for this article: J. Clin. Invest. 117:1049-1057 (2007). doi:10.1172/JCI30235
Stams et al. $(21,22)$ found that higher levels of ASNS expression were associated with greater resistance to asparaginase treatment in vitro and an inferior treatment outcome in some but not all genetic subtypes of ALL. It had been suggested that upregulation of ASNS in leukemic cells upon exposure to asparaginase may be the leading mechanism of resistance to the drug (18), but Appel et al. (23) recently found that upregulated ASNS expression did not correlate with a clinically poor response to the drug. Thus, the role of ASNS expression in leukemic cells with regard to asparaginase resistance may vary among genetic subtypes or, as suggested by others (24), it may have little or no relevance to drug sensitivity in vivo.

Leukemic lymphoblasts grow and accumulate in close association with bone marrow mesenchymal cells (MSCs). Direct contact with bone marrow MSCs is essential for the long-term survival and expansion of leukemic lymphoblasts in vitro (25-29), and their capacity to grow on MSCs is a predictor of treatment outcome in childhood ALL (30). We suspected that MSC niches might confer some degree of protection against asparaginase cytotoxicity. Here we demonstrated that bone marrow MSCs expressed high levels of ASNS, leading to increased concentrations of asparagine in the bone marrow microenvironment and subsequently protecting ALL blasts from the lethal action of asparaginase.

\section{Results}

ASNS expression in leukemic lymphoblasts and its relation to presenting characteristics. To determine the level of ASNS expression in leukemic lymphoblasts, we used a gene expression profiling database, obtained via Affymetrix U133A gene arrays, that included cell samples from 288 patients with newly diagnosed childhood ALL. The median signal intensity for ASNS (probe 205047_s_at) was 
Table 1

Correlation between ASNS expression with presenting clinicobiologic features

\begin{tabular}{|c|c|c|c|c|c|}
\hline \multirow[b]{2}{*}{$\begin{array}{l}\text { Presenting } \\
\text { feature }\end{array}$} & \multicolumn{5}{|c|}{ ASNS expression } \\
\hline & $\begin{array}{c}\text { No. of } \\
\text { patients }\end{array}$ & Lower & Middle & Higher & $P A$ \\
\hline \multicolumn{6}{|l|}{ Age (yr) } \\
\hline $\begin{array}{l}<1 \\
1-9 \\
\geq 10\end{array}$ & $\begin{array}{c}9 \\
202 \\
77\end{array}$ & $\begin{array}{c}3 \\
69 \\
24\end{array}$ & $\begin{array}{c}3 \\
69 \\
24\end{array}$ & $\begin{array}{c}3 \\
64 \\
29\end{array}$ & 0.9255 \\
\hline \multicolumn{6}{|l|}{ Race } \\
\hline $\begin{array}{l}\text { White } \\
\text { Black } \\
\text { Other }\end{array}$ & $\begin{array}{c}214 \\
55 \\
19\end{array}$ & $\begin{array}{c}78 \\
11 \\
7\end{array}$ & $\begin{array}{c}68 \\
24 \\
4\end{array}$ & $\begin{array}{c}68 \\
20 \\
8\end{array}$ & 0.1300 \\
\hline \multicolumn{6}{|l|}{ Gender } \\
\hline $\begin{array}{l}\text { Male } \\
\text { Female } \\
\text { wbc }(\times \mathbf{1 0} / \mathbf{l})\end{array}$ & $\begin{array}{l}169 \\
119\end{array}$ & $\begin{array}{l}48 \\
48\end{array}$ & $\begin{array}{l}62 \\
34\end{array}$ & $\begin{array}{l}59 \\
37\end{array}$ & 0.0977 \\
\hline $\begin{array}{l}<10 \\
10-50 \\
51-100 \\
>100,000\end{array}$ & $\begin{array}{l}91 \\
85 \\
52 \\
60\end{array}$ & $\begin{array}{l}29 \\
34 \\
17 \\
16\end{array}$ & $\begin{array}{l}30 \\
25 \\
21 \\
20\end{array}$ & $\begin{array}{l}32 \\
26 \\
14 \\
24\end{array}$ & 0.5596 \\
\hline \multicolumn{6}{|l|}{$B C R-A B L$} \\
\hline $\begin{array}{l}\text { Absent } \\
\text { Present }\end{array}$ & $\begin{array}{c}272 \\
16\end{array}$ & $\begin{array}{c}91 \\
5\end{array}$ & $\begin{array}{c}88 \\
8\end{array}$ & $\begin{array}{c}93 \\
3\end{array}$ & 0.2857 \\
\hline \multicolumn{6}{|l|}{$E 2 A-P B X 1$} \\
\hline $\begin{array}{l}\text { Absent } \\
\text { Present }\end{array}$ & $\begin{array}{c}266 \\
22\end{array}$ & $\begin{array}{c}92 \\
4\end{array}$ & $\begin{array}{l}85 \\
11\end{array}$ & $\begin{array}{c}89 \\
7\end{array}$ & 0.1629 \\
\hline \multicolumn{6}{|l|}{ TEL-AML1 } \\
\hline $\begin{array}{l}\text { Absent } \\
\text { Present } \\
\text { MLL-AF4 }\end{array}$ & $\begin{array}{c}231 \\
57\end{array}$ & $\begin{array}{l}69 \\
27\end{array}$ & $\begin{array}{l}79 \\
17\end{array}$ & $\begin{array}{l}83 \\
13\end{array}$ & 0.0034 \\
\hline $\begin{array}{l}\text { Absent } \\
\text { Present }\end{array}$ & $\begin{array}{c}270 \\
18\end{array}$ & $\begin{array}{c}91 \\
5\end{array}$ & $\begin{array}{c}89 \\
7\end{array}$ & $\begin{array}{c}90 \\
6\end{array}$ & 0.8376 \\
\hline \multicolumn{6}{|l|}{ Ploidy } \\
\hline $\begin{array}{l}\text { Hyperdiploid } \\
>50 \text { chromosomes } \\
\text { Other }\end{array}$ & $\begin{array}{c}51 \\
237\end{array}$ & $\begin{array}{l}33 \\
68\end{array}$ & $\begin{array}{l}11 \\
85\end{array}$ & $\begin{array}{c}7 \\
89\end{array}$ & $<0.0001$ \\
\hline \multicolumn{6}{|l|}{ Lineage } \\
\hline $\begin{array}{l}\text { T-lineage } \\
\text { B-lineage }\end{array}$ & $\begin{array}{c}47 \\
241\end{array}$ & $\begin{array}{c}6 \\
90\end{array}$ & $\begin{array}{l}15 \\
81\end{array}$ & $\begin{array}{l}26 \\
70\end{array}$ & 0.0005 \\
\hline \multicolumn{6}{|l|}{ DNA index } \\
\hline $\begin{array}{l}\geq 1.16 \\
\text { Other }\end{array}$ & $\begin{array}{c}47 \\
241\end{array}$ & $\begin{array}{l}27 \\
69\end{array}$ & $\begin{array}{l}13 \\
83\end{array}$ & $\begin{array}{c}7 \\
89\end{array}$ & 0.0003 \\
\hline
\end{tabular}

A total of 288 patients were studied; patients were assigned to equal groups of 96 by their ASNS expression levels as the lowest one-third, the middle one-third, and the highest one-third. ACalculated by general association test.

were more likely to have lower ASNS expression levels than were patients without these features $(P=0.033$ and $P<0.0001$, respectively). Similar relationships were observed when ASNS expression was analyzed as a continuous variable: higher ASNS levels in T-lineage ALL cases $(P<0.0001)$ and lower levels TEL-AML1 $(P=0.003)$ and hyperdiploid cases $(P<0.0001$; data not shown).

Expression of ASNS in MSCs. Leukemic lymphoblasts isolated from bone marrow rapidly die in standard culture media, but thrive on layers of bone marrow MSCs (25-30), suggesting their dependence on survival factors produced within this microenvironment. We therefore studied ASNS expression levels in bone marrow-derived MSCs. We first determined ASNS gene expression in bone marrow MSCs immortalized by enforced expression of the human telomerase reverse transcriptase (TERT) gene. These cells are indistinguishable from primary MSCs: they grow at a normal rate, they do not form tumors in immunodeficient mice, they can differentiate to osteoblasts and chondrocytes, and they support normal and leukemic hematopoiesis (31). The mean of 2 signal intensity measurements performed with the same gene array used to study ALL cells was 5,164.2, a value nearly 20 times higher than the median values determined in leukemic blasts (Figure 1A). We next used real-time PCR to compare ASNS expression in immortalized MSCs to that of primary MSCs (obtained from 9 healthy donors and 13 patients with ALL in complete remission) and primary leukemic cells obtained at diagnosis from 35 children with ALL. As shown in Figure 1B, ASNS gene expression levels in primary MSCs were variable but overall much higher than those measured in ALL samples. ASNS expression in primary MSCs was on average 23 times higher than in primary ALL cells. ASNS levels in 3 B-lineage ALL cell lines (RS4;11, 380, and REH) were higher than those of most primary ALL samples but lower than those of MSCs (Figure 1B). The cell line that most resemble primary ALL cells in terms of ASNS expression was RS4;11, as also shown by semiquantitative PCR and Western blotting (Figure 1, C and D). In contrast to what was previously reported for ALL cells $(20,32)$, we did not detect changes in ASNS expression by RT-PCR or Western blotting in MSCs exposed to asparaginase (1.0-5.0 IU/ml) for up to 48 hours (data not shown).

MSCs protect ALL cells from asparaginase cytotoxicity. To determine whether coculture with MSCs could affect the sensitivity of ALL cells to asparaginase,

265.4 (range, 8.8-2229.1). Comparison of ASNS expression levels (categorized as lower, middle, and higher) according to presenting clinicobiologic features (Table 1) failed to demonstrate significant relationships with most of these variables, including age and leukocyte count. However, expression levels were significantly higher in cases of T-lineage ALL compared with other subtypes $(P<0.0001)$. Within the B-lineage ALL group, patients with the TEL-AML1 gene or that were hyperdiploid (>50 chromosomes) we performed experiments with the 3 B-lineage ALL cell lines. For each cell line, we first determined the concentration of asparaginase that would cause $50 \%$ killing after 2 days of culture in cultures without MSCs. Then we used this concentration to treat the cell lines in parallel cultures performed with and without TERT-immortalized MSCs. As shown in Figure 2, the number of viable leukemic cells recovered after exposure to asparaginase for 2 days in 10 experiments with the 3 cell lines was consistently 
A
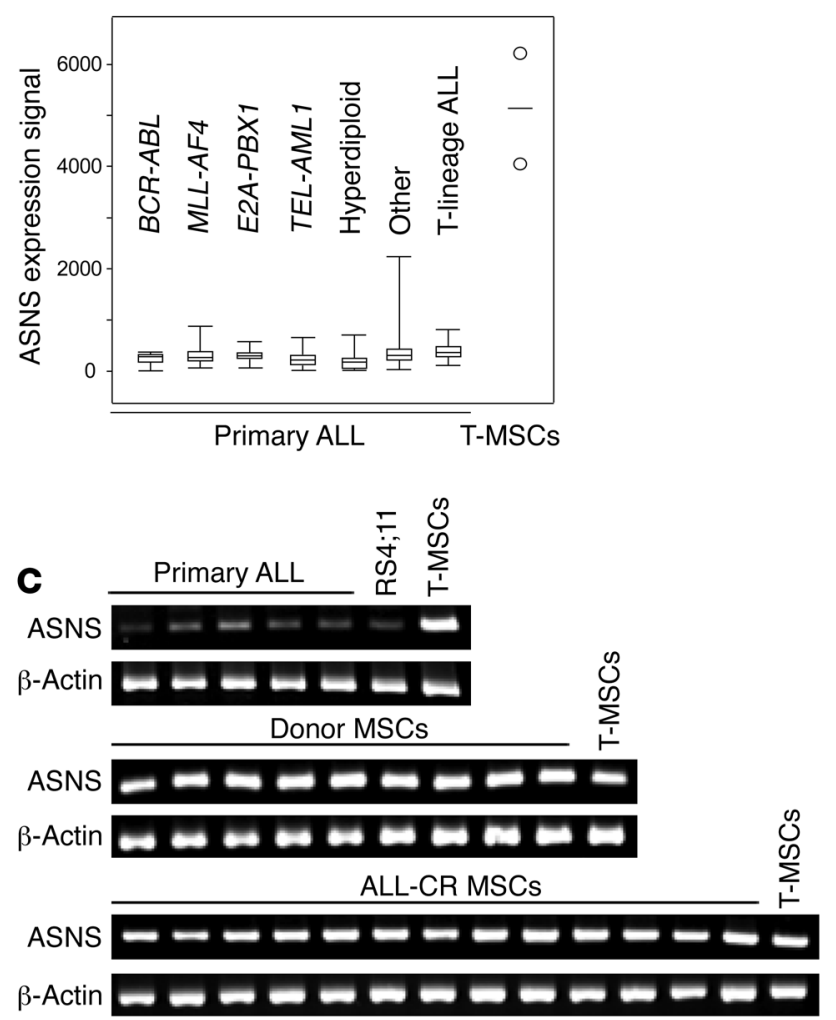

Figure 1

Expression of ASNS in ALL cells and bone marrow-derived MSCs. (A) Expression of ASNS mRNA in 288 samples of primary ALL cells compared with that of TERT-immortalized MSCs (T-MSCs; mean of 2 measurements denoted by circles) by Affymetrix GeneChip arrays. Boxes denote interquartile range; whiskers denote range; lines denote median. ${ }^{*}$ Cases lacking known genetic abnormalities. (B) Expression of ASNS mRNA in ALL cell lines, primary ALL samples and primary MSCs as measured by real-time RT-PCR. Primary MSCs were obtained from the bone marrow of 9 healthy donors (Donor MSCs) and 13 patients with ALL undergoing therapy and in complete remission (ALL-CR MSCs). Shown is ASNS expression relative to that of the TBP gene. (C) ASNS mRNA expression in primary ALL cell samples, the RS4;11 ALL cell line, and primary MSCs by semiquantitative RT-PCR; signals obtained with TERT-immortalized MSCs are also shown. $\beta$-Actin was used as a control. (D) Western blot analysis of ASNS protein expression in ALL cell lines and TERT-immortalized MSCs. After probing with the anti-ASNS antibody, the membrane was stripped and reprobed with an anti-actin antibody.

higher when cultures were performed in the presence of MSCs. To determine whether primary MSCs could also protect ALL cells from asparaginase cytotoxicity, we performed similar experiments using primary MSCs (obtained from 9 healthy individuals and 13 children with ALL in complete remission) instead of TERT-immortalized MSCs. As shown in Figure 2, primary MSCs also protected ALL cells from asparaginase cytotoxicity, regardless of whether they were obtained from ALL patients or healthy donors. There was no significant relation between protective capacity and MSCs levels of ASNS expression measured by realtime PCR (data not shown).

To ensure that the residual leukemic cells detected in cultures with MSCs were capable of proliferation, we performed experiments with the 3 cell lines in which we collected all residual cells after culture, washed out the asparaginase, and plated the cells in fresh medium in the absence of MSCs. After 7 days, the number of cells recovered from these cultures increased by 5 - to 21 -fold, indicating that the cells remaining on the MSC layers after asparaginase

B
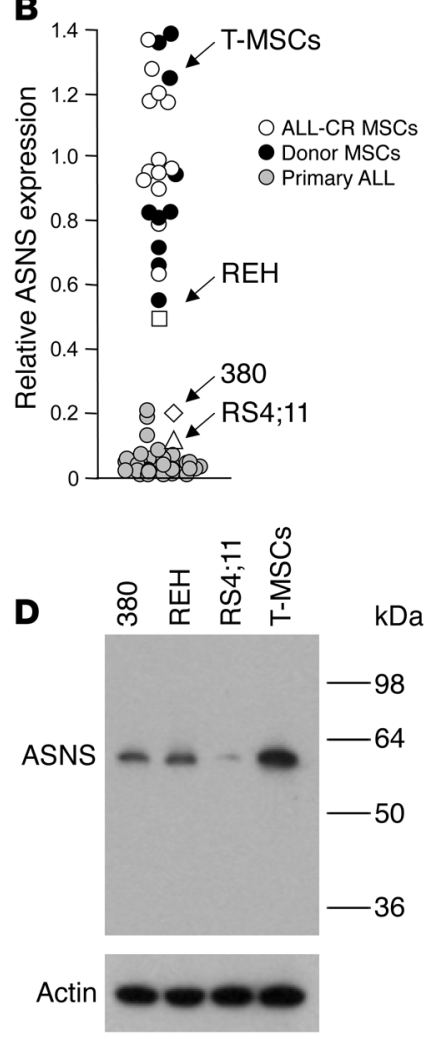

treatment were indeed viable and capable of proliferation. Reportedly, ASNS expression in ALL cells is upregulated after contact with mouse macrophages (33), but we did not detect increased levels of ASNS by Western blotting in RS4;11, 380, or REH cells after contact with MSCs (data not shown), ruling this out as the mechanism underlying the resistance of ALL cells to asparaginase that we observed.

Expression of ASNS in MSCs is associated with their capacity to support leukemic lymphoblasts. Taken together, the preceding results suggested that the protective effect of MSCs on ALL cells was related to their high levels of ASNS expression. To test this possibility, we decreased the levels of ASNS expression in immortalized MSCs using RNA interference and sought to determine whether this downregulation affected the capacity of the cells to protect ALL cells from asparaginase cytotoxicity. When the cells were transduced with a siRNA sequence targeted to ASNS, expression of the enzyme was markedly and stably decreased, whereas a scrambled sequence had no effect (Figure 3, $A$ and B). Although it lacked any effect on the rate of cell growth, morphology, or confluence pattern of MSCs, ASNS downregulation profoundly altered the cells' ability to protect ALL cells from asparaginase cytotoxicity. As shown in Figure 3C, asparaginase killed significantly more leukemic cells when the MSCs were transduced with the target siRNA sequence rather than the scrambled control sequence.

We next investigated whether enforced expression of ASNS in MSCs using a retroviral vector (Figure 4A) could augment their protective capacity. Although it did not produce changes in the growth rate or appearance of MSCs, stable ASNS overexpression significantly enhanced the cells' capacity to protect ALL cells from asparaginase cytotoxicity, as shown by experiments in which ALL cells were cultured on either MSCs overexpressing ASNS or cells transduced with a control vector lacking the ASNS gene (Figure 4B).

The third line of evidence supporting our central hypothesis came from studies with MSC clones defined by different levels of endogenous ASNS expression. These clones were derived from single-cell sorting of the TERT-immortalized MSCs used in the previous experiments. After screening the clones' ASNS expression, we identified one clone (referred to as clone B) that exhibited lower levels of ASNS (Figure 5A). Comparison of the protective capac- 

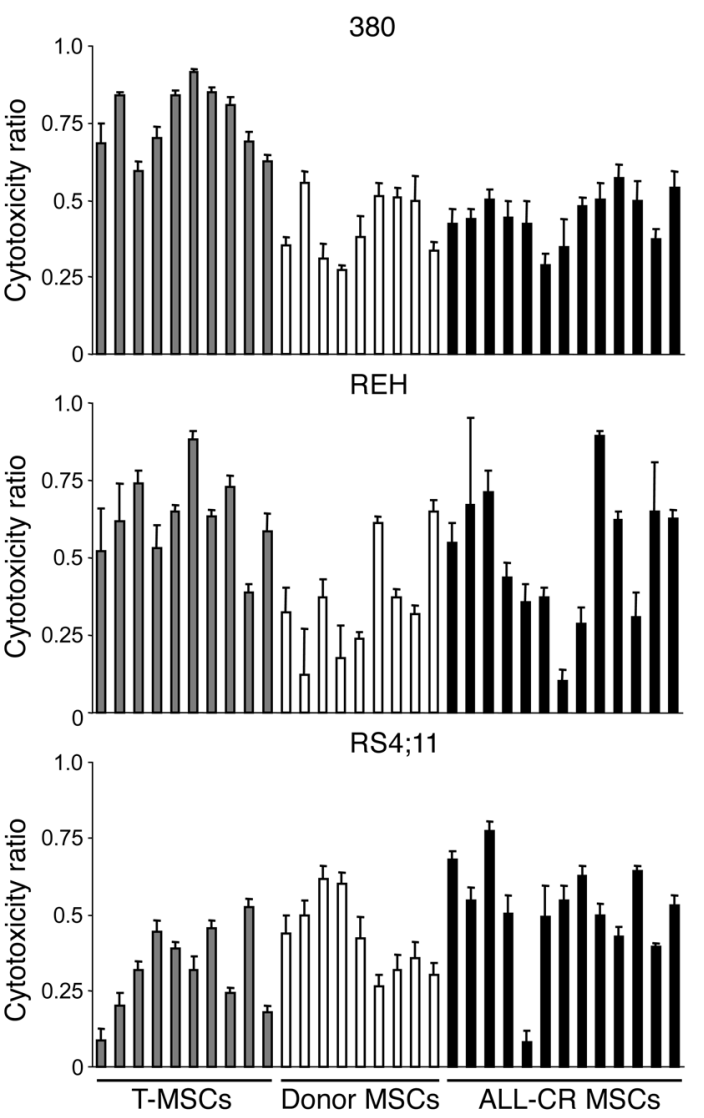

ity of this clone with clone A, whose ASNS expression level was similar to findings in unsorted MSCs, revealed a lower protective capacity for clone B (Figure 5B). We then asked whether increasing ASNS expression in clone $\mathrm{B}$ would restore its protective capacity. Transduction with the retroviral vector containing the ASNS construct described above led to robust and stable ASNS expression in the otherwise deficient MSCs (Figure 5A). Figure 5B shows the results of experiments comparing the protective capacity of the MSCs defective for endogenous ASNS after transduction with the ASNS-containing vector or an empty control vector: enforced expression of ASNS significantly improved the capacity of these MSCs to protect ALL cells from asparaginase cytotoxicity.

\section{Figure 3}

Downregulation of ASNS expression in MSCs by RNA interference decreases their capacity to protect ALL cells from asparaginase cytotoxicity. (A) Expression of ASNS mRNA in TERT-immortalized MSCs transduced with an ASNS-targeted siRNA construct (Tar) compared with that of cells transduced with a scrambled control construct (Scr) and that of nontransduced MSCs (Non) by semiquantitative RT-PCR. $\beta$-Actin was used as a control. (B) Expression of ASNS protein in the same cells by Western blotting. After probing with the anti-ASNS antibody, the membrane was stripped and reprobed with an anti-actin antibody. (C) Cytotoxicity of asparaginase against the ALL cell lines REH and RS4;11 was measured in coculture with MSCs transduced with the target siRNA construct or the scrambled control construct. Cytotoxicity was measured after 48 hours of exposure to $1 \mathrm{IU} / \mathrm{ml}$ and $0.001 \mathrm{IU} / \mathrm{ml}$ asparaginase for $\mathrm{REH}$ and $\mathrm{RS} 4 ; 11$, respectively. Each symbol indicates the results of 1 experiment (mean of 2 measurements). Bars correspond to the median of 8 experiments.
Figure 2

MSCs protect ALL cells from asparaginase cytotoxicity. The ALL cell lines 380, REH, and RS4;11 were cultured with and without MSCs for 48 hours in the presence of the concentration of asparaginase that causes $50 \%$ killing in 2 days of culture, as previously determined for each cell line in experiments without MSCs $(1.0 \mathrm{IU} / \mathrm{ml}$ for 380 and $\mathrm{REH} ; 0.001 \mathrm{IU} / \mathrm{ml}$ for RS4;11). Values are mean $\pm \mathrm{SD}$ of cell killing obtained in cultures with MSCs relative to that obtained in parallel cultures without MSCs. Ten experiments were performed for each ALL cell line with TERT-immortalized MSCs; MSCs from 9 healthy donors and 13 patients with ALL in complete remission were also tested for each ALL cell line. Each experiment consisted of 4 measurements.

Asparagine secretion by MSCs is determined by their expression of ASNS. Results of the previous experiments indicated that the mechanism underlying the protective capacity of MSCs relies on their high levels of ASNS expression. Since ASNS catalyzes asparagine biosynthesis, we postulated that MSCs mitigate the effect of asparaginase through the secretion of asparagine. In experiments with the RS4;11 cell line, addition of asparagine to the culture medium decreased the sensitivity of leukemic cells to asparaginase (Figure 6A). Measurement of the production of asparagine by MSCs showed that these cells do indeed secrete this amino acid in their milieu. Moreover, levels of asparagine in culture supernatants collected from MSCs after 24 hours of culture were directly related to levels of ASNS expression in the cells: in supernatants of MSCs treated with the siRNA target sequence, the amino acid was nearly undetectable (Figure 6B). In contrast, secretion of serine, glycine, and valine was not reduced by ASNS siRNA treatment (Figure 6B). In accord with these results, we found that culturing RS4;11 on a microporous membrane that prevents contact with MSCs but allows free flow of soluble factors also protected the cells from asparaginase when cultures were performed in the presence of MSCs (Figure 6C). Likewise, the addition of MSC-conditioned medium to cultures of RS4;11 cells reduced asparaginase cytotoxicity, whereas the addition of a mixture of MSC-derived cytokines - IL-1 $\alpha$, IL-1 $\beta$, IL-3, IL-6, IL-7, IL-11, stem cell factor, and Fms-like tyrosine kinase 3 ligand - instead of MSC-conditioned medium had no effect (Figure 6C).

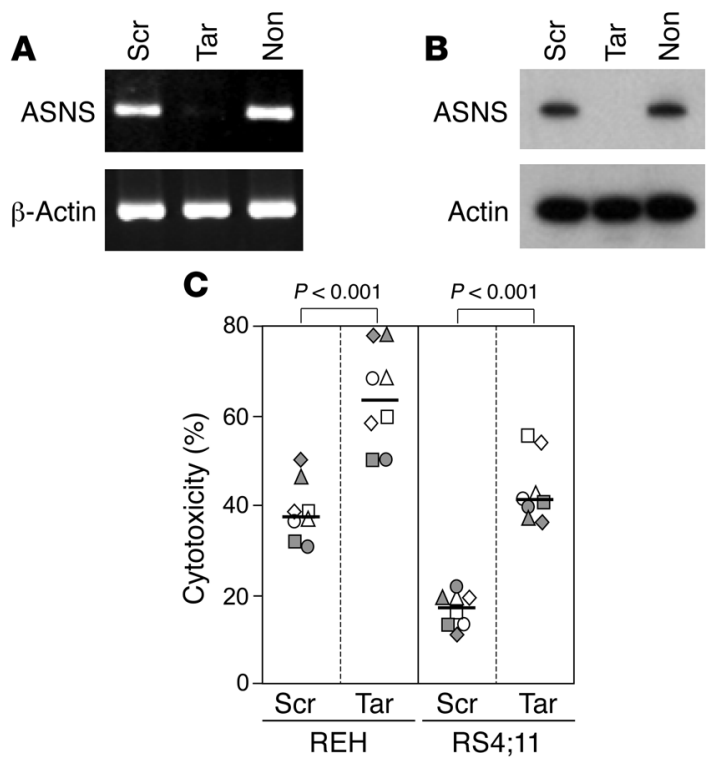


A

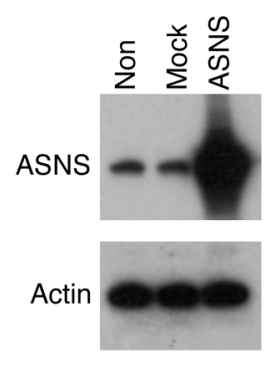

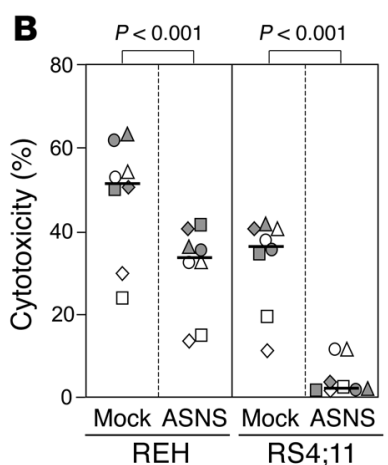

Figure 4

Upregulation of ASNS expression in MSCs increases their capacity to protect ALL cells from asparaginase cytotoxicity. (A) Expression of ASNS protein in TERT-immortalized MSCs transduced with an ASNS retroviral vector (ASNS) compared to that of cells transduced with an empty control vector (Mock) and that of nontransduced MSCs by Western blotting. After probing with the anti-ASNS antibody, the membrane was stripped and reprobed with an anti-actin antibody. (B) Cytotoxicity of asparaginase on the ALL cell lines REH and RS4;11 was measured in coculture with MSCs transduced with either ASNS or empty vector. Cytotoxicity was measured after 48 hours of exposure to $1 \mathrm{IU} / \mathrm{ml}$ and $0.001 \mathrm{IU} / \mathrm{ml}$ asparaginase for REH and RS4;11, respectively. Each symbol indicates the results of 1 experiment (mean of 2 measurements). Bars correspond to the median of 8 experiments.

Effect of MSCs on the sensitivity of primary ALL cells to asparaginase. We next tested whether the ASNS-dependent protective effects of MSCs on leukemic cell lines extend to primary ALL cells. In 28 of the 35 primary B-lineage ALL cases that we studied, asparaginase cytotoxicity when leukemic cells were cultured on MSCs with low ASNS expression levels due to siRNA treatment was significantly higher than that of cells transduced with a scrambled control construct $(P<0.05$; Figure 7A). Conversely, in 31 of the 35 cases, cytotoxicity was significantly decreased when the leukemic cells were cultured on MSCs overexpressing ASNS after retroviral transduction compared with cells transduced with an empty vector. These results indicate that ASNS expression in MSCs is an important determinant of the asparaginase sensitivity of primary ALL cells.

Using the combined data from the above experiments, we derived a MSC protection index in each case by subtracting the mean cytotoxicity ratio obtained in cultures with MSCs with upregulated
ASNS expression after transduction with the ASNS vector from that of MSC cultures in which ASNS expression was downregulated by siRNA. Figure 7B shows the relation between this index and the genetic subclassification of the 35 ALL cases studied. Of note, 4 of the 5 hyperdiploid ( $>50$ chromosomes) cases studied had an index below the median ( $P=0.068$, Wilcoxon rank-sum test), whereas 7 of 9 cases lacking known genetic abnormalities had an index above the median $(P=0.023)$. Susceptibility to the protective effects of MSCs was unrelated to the leukemic cells' immunophenotypic classification (early pre-B, pre-B, or transitional B; data not shown) or to the levels of ASNS expression in ALL cells as measured by gene expression array in 28 cases and by real-time PCR in all 35 cases (Figure 7C). We also assessed whether expression of amino acid transporter A1 and A2, 2 molecules that have previously been shown to participate in asparagine transport $(34,35)$, was related to ALL cell responses to the protective effect of MSCs. Transcripts encoding both molecules were detectable by microarray in all 28 cases studied, but there was no relation to leukemic cell responses to MSC protection (data not shown).

\section{Discussion}

Our study has revealed what we believe to be a novel mechanism for the resistance of ALL cells to asparaginase, one that relies on the interaction between these cells and the microenvironment in which they reside. When cultured in the presence of bone marrow-derived MSCs, leukemic cells were protected from asparaginase cytotoxicity, resulting in the persistence of viable leukemic cells capable of proliferation. This protective effect can be attributed to the higher expression of ASNS (which catalyzes the transfer of an amino group to aspartic acid to form asparagine) in MSCs than in leukemic lymphoblasts. Thus, MSCs with low expression of ASNS, either endogenous or downregulated by siRNA, failed to protect ALL cells from asparaginase cytoxicity, while enforced expression of the gene restored or enhanced this property. We also showed that ASNS expression in MSCs was linked to the synthesis and secretion of asparagine, the substrate of asparaginase, and that MSC-conditioned medium was sufficient to protect ALL cells from asparaginase, suggesting that MSCs exert their protective effect in vivo through secretion of asparagine into the bone marrow microenvironment. Importantly, the effect of MSCs on asparaginase-induced cytotoxicity was variable in this study, which suggests that this could be an important determinant of interpatient variability in response to the drug.

\section{Figure 5}

MSC clones with different levels of endogenous ASNS expression have different capacities to protect ALL cells from asparaginase cytotoxicity. (A) Different levels of expression of ASNS mRNA in TERT-immortalized unsorted MSCs and in 2 MSC clones (clones A and B) by semiquantitative RT-PCR. Expression of ASNS transcripts in clone B after retroviral transduction of ASNS or empty vector is also shown. $\beta$-Actin was used as a control. (B) Cytotoxicity of asparaginase against the ALL cell lines REH and RS4;11 was measured in coculture with MSCs with different levels of ASNS expression. Cytotoxicity was measured after 48 hours of exposure to $1 \mathrm{IU} / \mathrm{ml}$ and $0.001 \mathrm{IU} / \mathrm{ml}$ asparaginase for $\mathrm{REH}$ and RS4;11, respectively. Each symbol indicates the results of 1 experiment (mean of 2 measurements). Bars correspond to the median of 8 experiments.
A

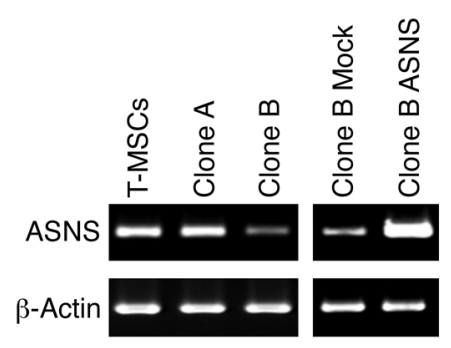

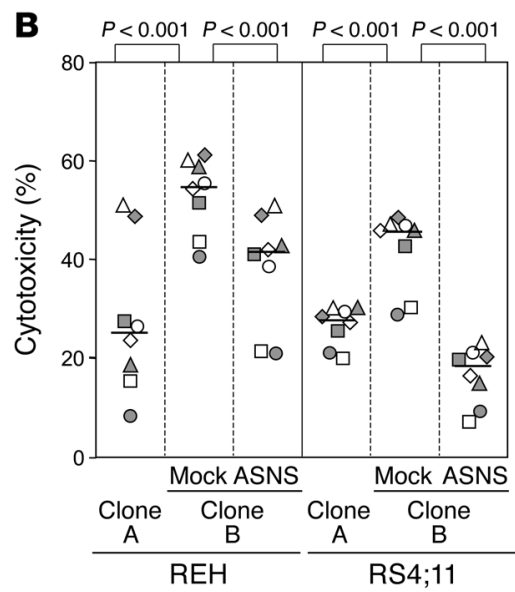


A

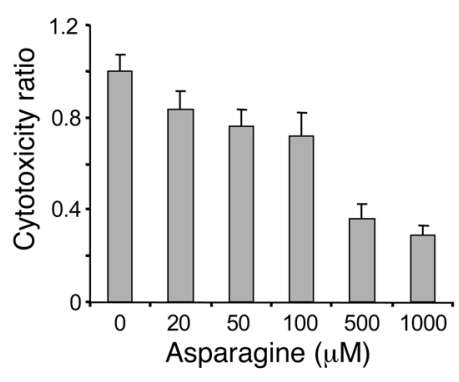

B
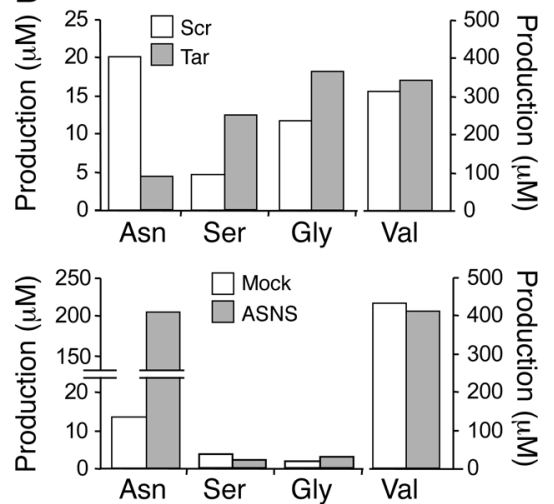

C

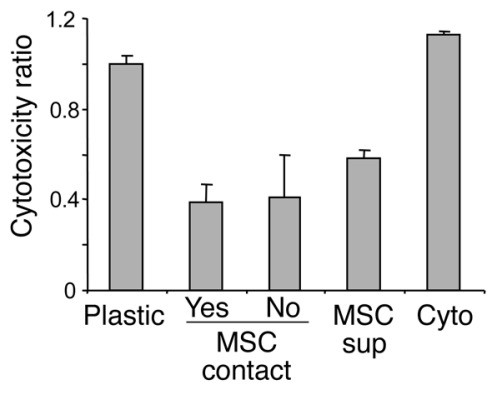

\section{Figure 6}

The protective effects of MSCs against asparaginase cytotoxicity are mediated by asparagine biosynthesis. (A) RS4;11 ALL cells were cultured for 48 hours in the presence of asparaginase $(0.001 \mathrm{IU} / \mathrm{ml})$. The effect of adding increasing concentrations of asparagine to the tissue culture medium are shown. Values are mean and SD of 4 measurements. (B) Concentration of asparagine in asparagine-free, FCS-free tissue culture medium (MEM) was measured after 24 hours of culture with MSCs expressing different levels of ASNS. Compared are MSCs with downregulated ASNS by siRNA and MSCs transduced with a scrambled sequence as well as MSCs with upregulated ASNS by retroviral transduction and MSCs transduced with an empty vector. Levels of control amino acids serine, glycine, and valine are also shown. (C) RS4;11 cells were cultured either in the absence of MSCs (Plastic), in direct contact with MSCs, in Transwell inserts suspended over MSCs (no MSC contact), in MSC-conditioned medium (collected after 48 hours of culture; MSC Sup) or with a mixture of MSC-derived cytokines (IL-1 $\alpha$, IL-1 $\beta$, IL-3, IL-6, IL-7, IL-11, stem cell factor, and Fms-like tyrosine kinase 3 ligand; Cyto). Cytotoxicity was measured after 48 hours of exposure to $0.001 \mathrm{IU} / \mathrm{ml}$ asparaginase. Values are mean and SD of 4 measurements.

Because of its preferential toxicity toward ALL cells, asparaginase has been widely used in combination chemotherapy for this disease for more than 3 decades (1-10), yet the molecular basis of asparaginase resistance remains poorly understood (16). Differences in susceptibility to asparaginase have long been attributed to the variable levels of ASNS expression in leukemic cells, but the results of more recent studies do not support this concept. For example, Fine et al. (32) reported that ASNS mRNA expression in ALL cells could not predict cellular responses to asparaginase in vitro. Likewise, Holleman et al. found that ASNS was not among the genes that best predicted resistance to asparaginase in vitro (36). Hence, the dogma that ASNS expression in ALL cells is the sole determinant of susceptibility to asparaginase has been challenged (24). Compared with ALL cells, MSCs express much higher levels of ASNS and continuously secrete asparagine, suggesting that resistance to asparaginase is mediated by MSCs rather than by endogenous ASNS expression in ALL cells. Although we observed differences in ASNS expression levels among primary MSC preparations, possibly due to functional polymorphisms in the ASNS gene, all MSC preparations tested were capable of protecting ALL cells from asparaginase cytotoxicity regardless of the former's ASNS expression. Conversely, we found that primary ALL cells varied markedly in their susceptibility to the protective effects of MSCs. These results indicate that inherent differences in the ability of leukemic cells to interact with the microenvironment account for different resistance to asparaginase.

In many biologic systems, the survival and growth of a cell population depends on factors provided by a nurturing microenvironment (37). For leukemic cells, this microenvironment is formed by MSCs of the bone marrow $(29,38-40)$ and may resemble the niches that sequester and regulate hematopoietic stem cells $(41,42)$. The results of our study indicate that this microenvironment can regulate the response of leukemic cells to chemotherapy. In support of this notion, Matsunaga et al. (43) reported that the attachment of acute myeloid leukemia cells to the bone marrow microenvironment through interaction between very late antigen-4 on leukemic cells and fibronectin on MSCs was crucial for the persistence of drug-resistant disease. Moreover, Mudry et al. (44) showed that signaling mediated through interaction with the MSC adhesion molecule VCAM-1 was required to maintain the maximum viability of ALL cells during exposure to cytarabine and etoposide, while Konopleva et al. (45) observed that MSCs upregulated the expression of antiapoptotic molecules and increased resistance to cytarabine in acute myeloid leukemia cells. We had previously observed that corticosteroid cytotoxicity against some ALL cell lines was diminished in the presence of MSCs (46). Taken together, these data suggest a scenario in which leukemic cells that reside in microenvironmental niches become relatively insensitive to chemotherapy. In the case of asparaginase, protection appears to be conferred by an environment rich in asparagine. If the cells residing in these privileged niches have stem cell properties, they might ultimately be capable of initiating leukemia recurrence.

Given the importance of asparaginase in ALL therapy, considerable efforts are being devoted to the development of ASNS inhibitors for clinical use (16). Our findings predict that it would be beneficial to test these inhibitors not only on ASNS in ALL cells, but also on the enzyme expressed in MSCs. We also suggest that the capacity of MSCs to secrete asparagine might be decreased by drugs known to affect their function and viability, such as taxol derivatives and vincristine $(47,48)$, or to downregulate ASNS transcripts, such as cytarabine (49). If so, this might lead to the design of more effective schedules of asparaginase administration. Finally, once the molecular mechanisms contributing to the interaction between leukemic cells and the bone marrow microenvironment are better understood, it may be possible to devise strategies that would alter it, thus enhancing the antileukemic action of asparaginase and perhaps other antileukemic agents as well. 
A
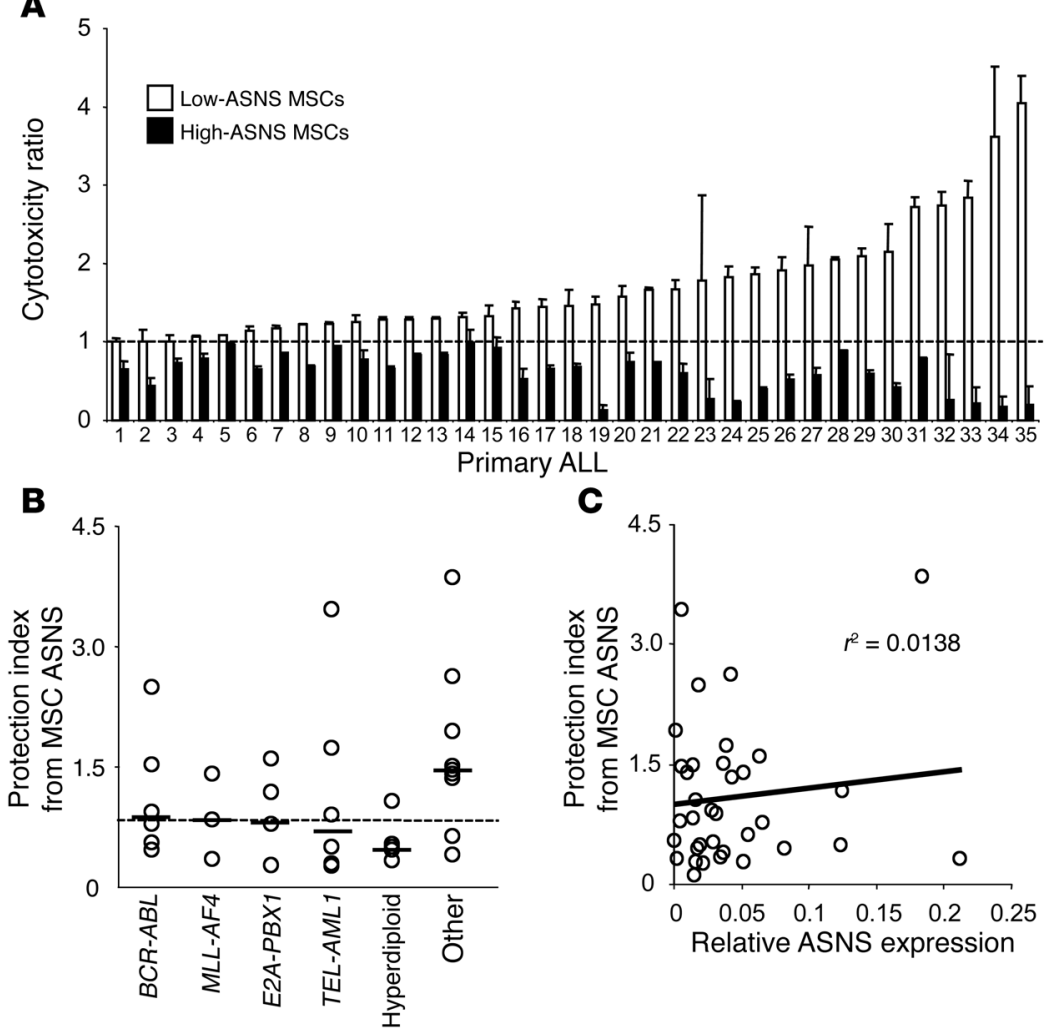

\section{Figure 7}

Expression of ASNS in MSCs determines the susceptibility of primary ALL cells to asparaginase. (A) Primary ALL cells, obtained from the diagnostic bone marrow of 35 children with ALL, were exposed for 48 hours to asparaginase $(1.0 \mathrm{lU} / \mathrm{ml})$ in the presence of MSCs with ASNS expression downregulated by siRNA or upregulated by ASNS transduction. Results were compared to those of parallel cultures using MSCs transduced with a scrambled siRNA construct or empty vector, respectively. Values are mean \pm SD cell killing obtained in cultures with each MSC type relative to that of its corresponding control (indicated by the dashed line). Differences between the test and control cultures were significant $(P<0.05$, Student's $t$ test) in all samples except $1-6$ and 23 in cultures with MSCs underexpressing ASNS and 5 and $14-16$ in cultures with MSCs overexpressing ASNS. (B) The mean cytotoxicity ratio obtained in cultures with MSCs overexpressing ASNS were subtracted from those with MSCs underexpressing ASNS to derive a protection index. Figure shows the relation between this protection index and genetic subtype in the 35 ALL samples. $P=0.068$, hyperdiploid cases; ${ }^{*}$ Cases lacking known genetic abnormalities, $P=0.023$; Wilcoxon rank-sum test. (C) Relationship between protection index and ASNS transcript expression in ALL cells by real-time PCR. $r^{2}$ by regression analysis is shown.

\section{Methods}

Cells. The human ALL cell lines - 380, with the $\mathrm{t}(8 ; 14 ; 18)(\mathrm{q} 24 ; \mathrm{q} 32 ; \mathrm{q} 21)$ abnormality; RS4;11, with the MLL-AF4 abnormality; and REH, with the TEL-AML1 abnormality - representative of childhood B-lineage ALL were obtained from Deutsche Sammlung von Mikroorganismen und Zellkulturen GmbH. Cells were maintained in RPMI-1640 (Invitrogen) with 10\% FCS (BioWhittaker Inc.) and antibiotics. Primary leukemia cells were obtained from patients with newly diagnosed B-lineage ALL. In all experiments, ALL samples were used after cryopreservation, and cell viability exceeded $85 \%$ by trypan blue dye exclusion. Mononuclear cells were obtained by density-gradient separation (Lymphoprep; Nycomed). MSCs were prepared from bone marrow mononuclear cells obtained from 9 healthy donors and 13 patients with ALL in complete morphologic remission, as previously described (29-31). Human MSCs immortalized by enforcing the expression of TERT in primary bone marrow MSCs were established in our laboratory (31). All primary cells were obtained with the approval of the St. Jude Children's Research Hospital Institutional Review Board and with appropriate informed consent.

Cell cultures and treatment with asparaginase. To test asparaginase cytotoxicity against ALL cells, we resuspended $2-5 \times 10^{4}$ cells from ALL cell lines or $2 \times 10^{5}$ primary ALL cells in RPMI- 1640 with $10 \%$ FCS. Cell suspensions $(200 \mu \mathrm{l})$ were placed in a 96 -well flat-bottomed plate with or without bone marrow-derived MSCs. In some experiments, ALL cells were cultured with MSC-conditioned medium, which was prepared by incubating MSCs in RPMI-1640 with 10\% FCS for 48 hours. Asparaginase (Elspar; Merck) was first dissolved in RPMI-1640 medium at 1,000 IU/ml and used at final concentrations ranging from $0.0001 \mathrm{IU} / \mathrm{ml}$ to $2.0 \mathrm{IU} / \mathrm{ml}$. L-asparagine (Sigma-Aldrich) was diluted in asparagine-free MEM (Invitrogen) before it was added to the cultures at the final concentrations indicated in Results. Recombinant human IL-1 $\alpha$, IL-1 $\beta$, IL-3, IL-6, IL-7, IL-11, stem cell factor, and Fms-like tyrosine kinase 3 ligand were purchased from R\&D Systems. All cell cultures were performed at $37^{\circ} \mathrm{C}$ in $5 \% \mathrm{CO}_{2}$.
Upon termination of the cultures, cells were harvested, labeled with PEconjugated anti-CD19 antibody, and assayed by a flow cytometric method specifically designed to enumerate leukemic cells recovered from culture, as previously described (47). Briefly, cells harvested from the cultures and labeled with anti-CD19 were resuspended in $0.5 \%$ paraformaldehyde, and the numbers of viable CD19+ ALL cells counted for each culture were compared.

Detection of ASNS expression. Levels of ASNS mRNA expression in leukemic cells were obtained from a database representing 288 cases of newly diagnosed childhood ALL that was generated at our institution by hybridizing leukemic cell cDNA to HG_U133A GeneChip arrays (Affymetrix Inc.; ref. 50; and our unpublished results).

For semiquantitative RT-PCR analysis, cDNA was obtained by reverse transcription of RNA with random hexamers and amplified using the following primers: ASNS sense, 5'-ATCAGGGGGCTTGGACTC-3' (bp 771-788); antisense, 5'-TCCTCCTCGGCTTTTTCA-3' (bp 1,166-1,149); human $\beta$-actin sense, $5^{\prime}$-ATCTGGCACCACACCTTCTACAATGAGCTGCG-3' (bp 253-284); antisense, 5'-CGTCATACTCCTGCTTGCTGATCCACATCTGC-3' (bp 1,090-1,249). The PCR amplification consisted of 27 cycles ( 25 for $\beta$-actin) of $94^{\circ} \mathrm{C}$ for 30 seconds, $56^{\circ} \mathrm{C}$ for 30 seconds, and $72^{\circ} \mathrm{C}$ for 60 seconds. PCR products were separated on a $2 \%$ agarose gel, and the DNA was visualized by ethidium bromide staining.

Real-time PCR was performed with ASNS-specific primers and an ABI Prism 7700 Sequence Detector (Applied Biosystems). Transcripts of the TATA box-binding protein (TBP) gene were used as a reference. The sequences of the primers for ASNS and TBP were as follows: ASNS forward, 5'-GCCCATGGTCTTGAACT-3' (bp 1,231-1,247); reverse, 5'-TTTGGTCGCCAGAGAAT-3' (bp 1,400-1,384); TBP forward, 5'CACGAACCACGGCACTGATT-3' (bp 620-639); reverse, 5' -TTTTCTTGCTGCCAGTCTGGAC-3' (bp 708-687). The sequences of TaqMan probes labeled with the reporter dye 6-carboxyfluorescein (FAM) and the quencher dye 6-carboxytetramethylrhodamine (TAMRA) were FAM-CTT- 
GTCTCTGCCACCAGAAATGA-(bp 1,287-1,309)-TAMRA for ASNS and FAM-TGTGCACAGGAGCCAAGAGTGAAGA-(bp 659-683)-TAMRA for TBP. For PCR we performed 1 cycle of $50^{\circ} \mathrm{C}$ for 2 minutes, 1 cycle of $95^{\circ} \mathrm{C}$ for 10 minutes, 40 cycles of $95^{\circ} \mathrm{C}$ for 15 seconds, and 1 cycle of $60^{\circ} \mathrm{C}$ for 1 minute. After determining the threshold cycle (Ct) for ASNS and TBP, we calculated the expression of ASNS as 2-(CtASNS-CtTBP).

For Western blotting, cells were washed with PBS, and the pellet was resuspended in lysis buffer consisting of PBS, $1 \%$ Triton-X 100, $0.5 \%$ sodium deoxycholate, $0.1 \% \mathrm{SDS}, 3 \mu \mathrm{g} / \mathrm{ml}$ pepstatin, $3 \mu \mathrm{g} / \mathrm{ml}$ leupeptin, $1 \mathrm{mM}$ PMSF, $2 \mathrm{mM}$ EDTA, and $5 \mu \mathrm{g} / \mathrm{ml}$ aprotinin. Cell lysates were separated by SDS-PAGE on a $10 \%-20 \%$ duramide gel (Cambrex). After transfer to a polyvinylidene difluoride membrane, this was incubated with an antiASNS monoclonal antibody (gift from M.S. Kilberg, University of Florida, Gainesville, Florida, USA; ref. 19) and then with a goat anti-mouse IgG horseradish peroxidase-conjugated antibody. Antibody binding was detected with the ECL kit (Pharmacia).

Generation of MSCs with different levels of ASNS expression. To downregulate ASNS expression in MSCs, we used stably expressed siRNA. The ASNS target construct was inserted into the pSUPER.retro.neo.gfp plasmid (OligoEngine) with the use of specific oligonucleotides (denoted by underline; bp 422-440) as follows: forward, GATCCCCGAGTCAGACCTTTGTTTAATTCAAGAGATTAAACAAAGGTCTGACTCTTTTTGGAAA; reverse, AGCTTTTCCAAAAAGAGTCAGACCTTTGTTTAATCTCTTGAATTAAACAAAGGTCTGACTCGGG. We also made a scrambled vector using a 19-nucleotide sequence (GTAATCTCAGTGCATTTAG) that lacked significant homology to any mammalian gene sequence and used it as a nonsilencing control. Oligonucleotides were obtained from the St. Jude Hartwell Center. To generate viral supernatant, we used FuGENE 6 (Roche Applied Science) to transfect $3 \times 10^{6} 293 \mathrm{~T}$ cells and maintained them in $10-\mathrm{cm}$ tissue culture dishes (Falcon; BD) for 24 hours with $3.5 \mu \mathrm{g}$ vectors, $3.5 \mu \mathrm{g}$ helper plasmid pEQ-PAM3(-E), and $3.5 \mu \mathrm{g}$ envelope plasmid pRDF. After 24 hours, the medium was replaced with RPMI-1640 containing $10 \%$ FCS and antibiotics. Conditioned medium containing retrovirus was harvested 48 and 72 hours after transfection, immediately frozen in dry ice, and stored at $-80^{\circ} \mathrm{C}$ until use. HeLa cells were used to titrate virus concentration. The viral supernatant containing the pSUPER target or scrambled sequence was used to infect MSCs. Retroviral infections were performed in the presence of $4 \mu \mathrm{g} / \mathrm{ml}$ polybrene (Sigma-Aldrich). After 72 hours, retrovirally infected cells were selected with $0.15 \mu \mathrm{g} / \mu \mathrm{l}$ G418 (Geneticin; Sigma-Aldrich), and bulk cells were used for the experiments.

To enforce the expression of ASNS in MSCs, we used retroviral transduction with a murine stem cell virus-internal ribosome entry siteGFP (MSCV-IRES-GFP) vector obtained from the St. Jude Vector Development and Production Shared Resource. Human ASNS tagged with a flag peptide (DYKDDDDK) at the $C$ terminus was subcloned into the BstBI and MluI sites of the MSCV-IRES-GFP vector by PCR, using human MSC cDNA as a template. The viral supernatant (produced as described above and containing MSCV-ASNS-IRES-GFP) was used to infect immortalized MSCs. After transduction of MSCV-ASNS-IRES-GFP, the transduced cells were sorted by detection of GFP expression with use of a fluorescence-activated cell sorter (MoFlo; Cytomation Inc.).

Amino acid analysis. To determine the production of asparagine and other amino acids by MSCs, we cultured $2 \times 10^{4}$ cells into 96 -well plates. After the layers reached confluency, the medium was replaced by asparagine-free MEM medium supplemented with L-glutamine at $37^{\circ} \mathrm{C}$ in $5 \% \mathrm{CO}_{2}$. After 24 hours, the supernatants were collected, placed into acid-washed tubes, and stored at $-80^{\circ} \mathrm{C}$ until use. The amino acids in supernatants were analyzed by Commonwealth Biotechnologies Inc. The amino acids were subjected to precolumn derivatization with ortho-phthalaldehyde for the primary amino acids and 9-fluorenylmethyl-chloroformate for the secondary amino acids. The derivatized amino acids were separated on a reverse-phase C-18 column and detected by diode-array detection. Calibration and quantitation of peaks were done by using Amino Quant software (Agilent Technologies).

Statistics. We used the general association test to relate levels of ASNS expression (measured by microarray) to clinicobiologic features of ALL patients and to early treatment response. Wilcoxon rank-sum tests or paired 2-tailed or 2-sample Student's $t$ tests were used to determine the significance of differences observed between the results of asparaginase cytotoxicity testing. A $P$ value less than 0.05 was considered statistically significant.

\section{Acknowledgments}

We thank Michael Kilberg for providing the anti-ASNS monoclonal antibody and for valuable comments, Pat Stow for advice with real-time PCR, Deqing Pei and Stanley Pounds for statistical analysis, Mary Relling and Lisa Trevino for helpful information, and Elaine Coustan-Smith and the St. Jude Tissue Resource Laboratory for providing bone marrow and leukemia samples. This work was supported by grants CA58297, CA60419, and CA21765 from the National Cancer Institute, NIH, and by the American Lebanese Syrian Associated Charities (ALSAC).

Received for publication September 4, 2006, and accepted in revised form January 30, 2007.

Address correspondence to: Dario Campana, Department of Oncology, St. Jude Children's Research Hospital, 332 North Lauderdale Street, Memphis, Tennessee 38105, USA. Phone: (901) 495-2528; Fax: (901) 495-5947; E-mail: dario.campana@stjude.org.
1. Oettgen, H.F., et al. 1967. Inhibition of leukemias in man by L-asparaginase. Cancer Res. 27:2619-2631.

2. Tallal, L., et al. 1970. E. coli L-asparaginase in the treatment of leukemia and solid tumors in 131 children. Cancer. 25:306-320.

3. Pratt, C.B., Simone, J.V., Zee, P., Aur, R.J., and Johnson, W.W. 1970. Comparison of daily versus weekly L-asparaginase for the treatment of childhood acute leukemia. J. Pediatr. 77:474-483.

4. Jaffe, N., et al. 1971. L-asparaginase in the treatment of neoplastic diseases in children. Cancer Res. 31:942-949.

5. Sutow, W.W., et al. 1971. L-asparaginase therapy in children with advanced leukemia. The Southwest Cancer Chemotherapy Study Group. Cancer. 28:819-824.

6. Ortega, J.A., et al. 1977. L-Asparaginase, vincristine, and prednisone for induction of first remission in acute lymphocytic leukemia. Cancer Res. 37:535-540.
7. Sallan, S.E., et al. 1983. Influence of intensive asparaginase in the treatment of childhood nonT-cell acute lymphoblastic leukemia. Cancer Res. 43:5601-5607.

8. Reaman, G.H., Ladisch, S., Echelberger, C., and Poplack, D.G. 1980. Improved treatment results in the management of single and multiple relapses of acute lymphoblastic leukemia. Cancer. 45:3090-3094.

9. Pui, C.H., and Evans, W.E. 2006. Treatment of acute lymphoblastic leukemia. N. Engl. J. Med. 354:166-178.

10. Schrappe, M., et al. 2000. Improved outcome in childhood acute lymphoblastic leukemia despite reduced use of anthracyclines and cranial radiotherapy: results of trial ALL-BFM 90. GermanAustrian-Swiss ALL-BFM Study Group. Blood. 95:3310-3322.

11. Silverman, L.B., et al. 2001. Improved outcome for children with acute lymphoblastic leukemia: results of Dana-Farber Consortium Protocol 91-01.
Blood. 97:1211-1218.

12. Duval, M., et al. 2002. Comparison of Escherichia coli-asparaginase with Erwinia-asparaginase in the treatment of childhood lymphoid malignancies: results of a randomized European Organisation for Research and Treatment of Cancer-Children's Leukemia Group phase 3 trial. Blood. 99:2734-2739.

13. Pession, A., et al. 2005. Long-term results of a randomized trial on extended use of high dose L-asparaginase for standard risk childhood acute lymphoblastic leukemia. J. Clin. Oncol. 23:7161-7167.

14. Whitecar, J.P., Jr., Bodey, G.P., Harris, J.E., and Freireich, E.J. 1970. L-asparaginase. N. Engl. J. Med. 282:732-734.

15. Iiboshi, Y., Papst, P.J., Hunger, S.P., and Terada, N. 1999. L-Asparaginase inhibits the rapamycin-targeted signaling pathway. Biochem. Biophys. Res. Commun. 260:534-539.

16. Richards, N.G., and Kilberg, M.S. 2006. Asparagine synthetase chemotherapy. Annu. Rev. Biochem. 
75:629-654.

17. Prager, M.D., and Bachynsky, N. 1968. Asparagine synthetase in asparaginase resistant and susceptible mouse lymphomas. Biochem. Biophys. Res. Commun. 31:43-47.

18. Haskell, C.M., and Canellos, G.P. 1969. 1-asparaginase resistance in human leukemia--asparagine synthetase. Biochem. Pharmacol. 18:2578-2580.

19. Hutson, R.G., et al. 1997. Amino acid control of asparagine synthetase: relation to asparaginase resistance in human leukemia cells. Am. J. Physiol. 272:C1691-C1699.

20. Aslanian, A.M., Fletcher, B.S., and Kilberg, M.S. 2001. Asparagine synthetase expression alone is sufficient to induce l-asparaginase resistance in MOLT-4 human leukaemia cells. Biochem. J. 357:321-328.

21. Stams, W.A., et al. 2005. Asparagine synthetase expression is linked with L-asparaginase resistance in TEL-AML1-negative but not TEL-AML1-positive pediatric acute lymphoblastic leukemia. Blood. 105:4223-4225.

22. Stams, W.A., et al. 2003. Sensitivity to L-asparaginase is not associated with expression levels of asparagine synthetase in $\mathrm{t}(12 ; 21)+$ pediatric ALL. Blood. 101:2743-2747.

23. Appel, I.M., et al. 2006. Upregulation of asparagine synthetase expression is not linked to the clinical response to L-asparaginase in pediatric acute lymphoblastic leukemia. Blood. 107:4244-4249.

24. den Boer, M.L., Evans, W.E., and Pieters, R. 2005. TELAML1-positive ALL: a discordant genotype. Cell Cycle. 4:997-998.

25. Umiel, T., et al. 1986. Long-term culture of infant leukemia cells: dependence upon stromal cells from the bone marrow and bilineage differentiation. Leuk. Res. 10:1007-1013.

26. Gluck, U., et al. 1989. Long-term proliferation of human leukemia cells induced by mouse stroma. Exp. Hematol. 17:398-404.

27. Manabe, A., Coustan-Smith, E., Behm, F.G., Raimondi, S.C., and Campana, D. 1992. Bone marrow-derived stromal cells prevent apoptotic cell death in B-lineage acute lymphoblastic leukemia. Blood. 79:2370-2377.

28. Makrynikola, V., and Bradstock, K.F. 1993. Adhesion of precursor-B acute lymphoblastic leukaemia cells to bone marrow stromal proteins. Leukemia. 7:86-92.

29. Nishigaki, H., et al. 1997. Prevalence and growth characteristics of malignant stem cells in Blineage acute lymphoblastic leukemia. Blood. 89:3735-3744.

30. Kumagai, M., et al. 1996. Stroma-supported culture in childhood B-lineage acute lymphoblastic leukemia cells predicts treatment outcome. J. Clin. Invest. 97:755-760.

31. Mihara, K., et al. 2003. Development and functional characterization of human bone marrow mesenchymal cells immortalized by enforced expression of telomerase. Br. J. Haematol. 120:846-849.

32. Fine, B.M., Kaspers, G.J., Ho, M., Loonen, A.H., and Boxer, L.M. 2005. A genome-wide view of the in vitro response to l-asparaginase in acute lymphoblastic leukemia. Cancer Res. 65:291-299.

33. Ding, Y., Li, Z., and Broome, J.D. 2005. Epigenetic changes in the repression and induction of asparagine synthetase in human leukemic cell lines. Leukemia. 19:420-426.

34. Wang, H., et al. 2000. Cloning and functional expression of ATA1, a subtype of amino acid transporter A, from human placenta. Biochem. Biophys. Res. Commun. 273:1175-1179.

35. Hatanaka, T., et al. 2000. Primary structure, functional characteristics and tissue expression pattern of human ATA2, a subtype of amino acid transport system A. Biochim. Biophys. Acta. 1467:1-6.

36. Holleman, A., et al. 2004. Gene-expression patterns in drug-resistant acute lymphoblastic leukemia cells and response to treatment. N. Engl. J. Med. 351:533-542.

37. Raff, M.C. 1992. Social controls on cell survival and cell death. Nature. 356:397-400.

38. Manabe, A., et al. 1994. Adhesion-dependent survival of normal and leukemic human B lymphoblasts on bone marrow stromal cells. Blood.
83:758-766.

39. Bradstock, K., Bianchi, A., Makrynikola, V., Filshie, R., and Gottlieb, D. 1996. Long-term survival and proliferation of precursor-B acute lymphoblastic leukemia cells on human bone marrow stroma. Lenkemia. 10:813-820.

40. LeBien, T.W. 2000. Fates of human B-cell precursors. Blood. 96:9-23.

41. Scadden, D.T. 2006. The stem-cell niche as an entity of action. Nature. 441:1075-1079.

42. Moore, K.A., and Lemischka, I.R. 2006. Stem cells and their niches. Science. 311:1880-1885.

43. Matsunaga, T., et al. 2003. Interaction between leukemic-cell VLA-4 and stromal fibronectin is a decisive factor for minimal residual disease of acute myelogenous leukemia. Nat. Med. 9:1158-1165.

44. Mudry, R.E., Fortney, J.E., York, T., Hall, B.M., and Gibson, L.F. 2000. Stromal cells regulate survival of B-lineage leukemic cells during chemotherapy. Blood. 96:1926-1932.

45. Konopleva, M., et al. 2002. Stromal cells prevent apoptosis of AML cells by up-regulation of antiapoptotic proteins. Lenkemia. 16:1713-1724.

46. Ito, C., et al. 1996. Comparative cytotoxicity of dexamethasone and prednisolone in childhood acute lymphoblastic leukemia. J. Clin. Oncol. 14:2370-2376.

47. Campana, D., Manabe, A., and Evans, W.E. 1993. Stroma-supported immunocytometric assay (SIA): a novel method for testing the sensitivity of acute lymphoblastic leukemia cells to cytotoxic drugs. Lenkemia. 7:482-488.

48. Li, J., Law, H.K., Lau, Y.L., and Chan, G.C. 2004. Differential damage and recovery of human mesenchymal stem cells after exposure to chemotherapeutic agents. Br. J. Haematol. 127:326-334.

49. Takagaki, K., et al. 2003. cDNA microarray analysis of altered gene expression in Ara-C-treated leukemia cells. Biochem. Biophys. Res. Commun. 309:351-358.

50. Ross, M.E., et al. 2003. Classification of pediatric acute lymphoblastic leukemia by gene expression profiling. Blood. 102:2951-2959. 